\title{
INVENTAIRE 1980 DES CHIRONOMIDES (DIPTERA) CONNUS DES PYRENEES
}

\author{
par H. LAVILLE1
}

Un inventaire des Chironomides pyrénéens est présenté à la lumière de récoltes inédites sur le versant français: soit, des rivières Nivelle et Lissuraga dans l'ouest de la chaîne, de la Créssonnière d'Agos et du lac d'Orédon et de ses tributaires dans le Centre, et de la rivière Lladure dans l'Est. L'étude de ce matériel (Imagos $\sigma^{*}$ et Nymphes) permet de porter à 235 le nombre de chironomides recensés dans les Pyrénées. Il s'agit, pour 89 espèces $(+)$ de premières citations dans les Pyrénées et pour 23 d'entre elles $(++)$ de premières récoltes en France.

\section{0 - survey of the chironomids (Diptera) known from the Pyrenees.}

A list of Pyrenean chironomids is presented in the light of unpublished records from the French side of the mountain range: the rivers Nivelle and Lissuraga are in the west of the mountain chain, the Créssonnière of Agos and lake Orédon with ist tributaries in the centre, and the river Lladure in the East. The study of this material (adult male and pupae) brings to 235 the number of chironomid species taken in the Pyrenees. 89 species $(+)$ are first records for the Pyrenees and 23 of these species $(++)$ are first records for France.

\section{I. - INTRODUCTION}

Un premier inventaire des Chironomides pyrénéens ${ }^{2}$ présenté au 6: Symposium international de Prague en août 1976 et dont les actes devaient être publiés dans la revue tchèque "Acta Universitatis Carolinae, Ser. Biologica » (Prague), n'est pas paru à ce jour.

Un double du manuscrit, confié aux $D^{\text {rs }}$ Fittkau et Reiss (Munich) a servi de référence pour établir la liste des Chironomides signalés dans la zone 2 de la deuxième édition de la Limnofauna Europaea (Fittkau et al 1978). Mais, pour plusieurs espèces, les stations originales de récolte restent encore inédites. C'est pourquoi il nous semble intéressant de présenter ce deuxième catalogue remis à jour et complété par quelques récoltes plus récentes.

Depuis 1976, plusieurs travaux ont paru se rapportant à la faune des Chironomides pyrénéens. L'un concerne les Chironomides d'un torrent de haute montagne des Pyrénées centrales, l'Estaragne où

1. Laboratoire d'Hydrobiologie (ERA 702 du C.N.R.S.), Université Paul-Sabatier, 118, route de Narbonne, 31062 Toulouse Cedex, France.

2. "Inventaire des Chironomides connus des Pyrénées en 1976 ". 


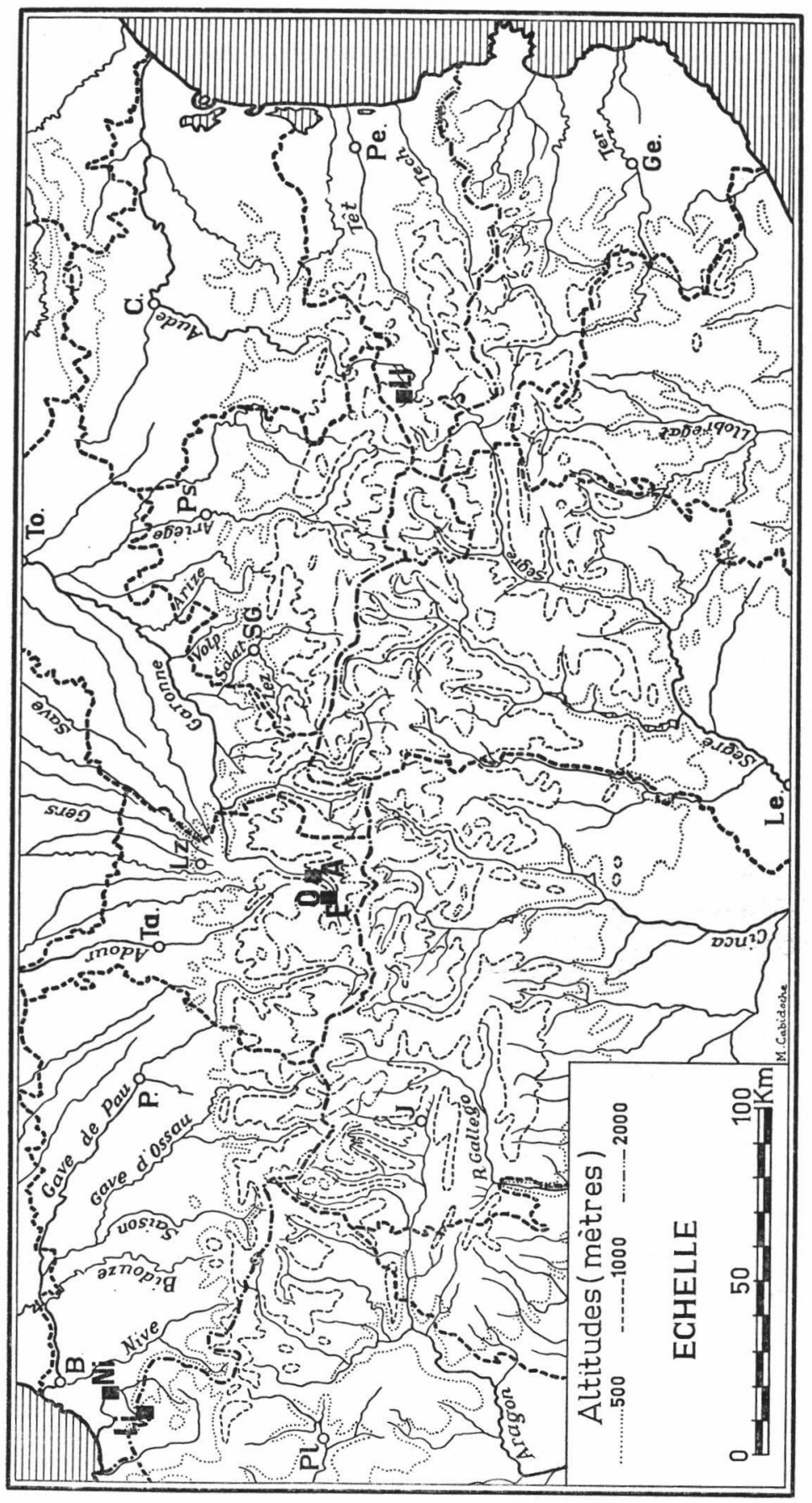

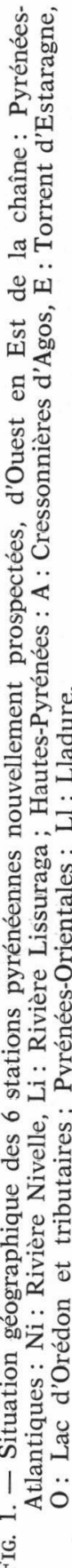


61 espèces sont recensées (Laville et Lavandier 1977). Un autre porte sur les Chironomides de Catalogne espagnole où 34 espèces sont signalées (Prat 1977). On trouve enfin quelques citations dans un travail récent paru en deux parties : la première (Prat 1979) signale 2 Tanypodinae et 3 Orthocladiinae dans trois lacs de barrage catalans, la seconde (Prat 1980) note la récolte de 3 Chironominae dans ces mêmes lacs.

\section{II. - LOCALISATION DES RECOLTES INEDITES}

Les stations pyrénéennes dont les récoltes sont encore inédites intéressent le versant français : elles ont souvent fait l'objet d'un chapitre dans des travaux de $3^{e}$ cycle du Laboratoire d'Hydrobiologie de Touiouse : il s'agit de la Cressonnière d'Agos (Dumas et Lavandier 1970), du lac d'Orédon et de ses tributaires (Masson 1977) dans les Pyrénées centrales; de la rivière Lladure dans les Pyrénées orientales (Pujol 1975) ${ }^{1}$. D'autres récoltes dans les rivières Nivelle et Lissuraga (Pyrénées atlantiques) n'ont jamais été pub!liées (Neveu, in litt.)².

Stations nouvelles :

A) Pyrénées atlantiques

1. Pisciculture de Saint-Pée-sur-Nivelle. Petit fleuve (alt. $30 \mathrm{~m}$ ) du littoral atlantique aux pieds des Pyrénées : récoltes au piège lumineux ; déterminations A. Neveu.

2. Rivière le Lissuraga $(7 \mathrm{~km})$ : coule de $400 \mathrm{~m}$ à $48 \mathrm{~m}$ où il se jette dans le Lourgorietta lui-même affluent de la Nivelle (Thibault 1971, Neveu 1972). Récoltes au piège à émergence et au filet à dérive; déterminations A. Neveu.

B) Pyrénées centrales : massif de Néouvielle

1. Cressonnière d'Agos.

Trois ruisseaux et deux mares sur la rive gauche de la Neste d'Aure près du village d'Agos $(780 \mathrm{~m})$, département des Hautes-Pyrénées. Présentation et caractéristiques physico-chimiques des milieux par Dumas et Lavandier (1970). Imagos capturés en vol ou obtenus d'élevages : récoltes J. Dumas et P. Lavandier en 1968-69: déterminations H. Laville.

1. La liste des Chironomides de la Lladure n'est pas incluse dans ce travail: elle m'a été communiquée par J.-N. Tourenq (Toulouse) que je remercie bien sincèrement.

2. Je remercie A. Neveu (Rennes) qui m'a obligeamment autorisé à insérer dans ce catalogue la liste des Chironomides de ces deux rivières. 
2. Torrent d'Estaragne.

Torrent de haute montagne (2 350 à $1850 \mathrm{~m}$ ), l'Estarragne, long de $3 \mathrm{~km}$ environ, se jette dans le lac d'Orédon. Les caractéristiques physiques (Lavandier 1974) et chimiques (Lavandier et Mur 1974) sont analysées en relation avec les conditions géographiques générales. Les récoltes d'imagos ont été obtenues par piégeages, chasses en vol, les nymphes et exuvies nymphales obtenues par filets à dérive : récoltes $P$. Lavandier ; détermination $\mathbf{H}$. Laville. Liste faunistique de Laville et Lavandier (1977).

3. Lac d'Orédon $(1850 \mathrm{~m})$ et sources rhéocrènes tributaires.

Récoltes sur substrats artificiels flottants dans la zone littorale en juillet, août et septembre 1976. Faune lacustre (9 espèces) et lotique (29 espèces) (Masson 1977 : déterminations H. Laville et J.-N. Tourenq).

\section{C) Pyrénées orientales}

Rivière la Lladure (1 600-1 400 m). Premier affluent de l'Aude. Présentation du milieu (Pujol 1975). Récoltes en vol et au filet de Surber: J.-Y. Pujol ; déterminations J.-N. Tourenq.

\section{III. - PRESENTATION DES DONNEES}

L'inventaire faunistique ci-après a été remanié à la lumière des travaux postérieurs à 1976 et la nomenclature systématique utilisée suit celle de la Limnofauna europaea (Fittkau et al 1978).

Cet inventaire est présenté sous forme de tableau : la première colonne (Z2) reprend les citations de la zone 2 de la Limnofauna. Les colonnes suivantes indiquent les récoltes inédites des différentes stations ou, pour ce qui concerne l'Estaragne, redonnent la liste de Laville et Lavandier (1977). Soit, d'Ouest en Est de la chaîne : $\mathrm{Ni}=$ Nivelle ; $\mathrm{Li}=$ Lissuraga; $\mathrm{A}=$ Agos $; \mathrm{E}=$ Estaragne; $\mathrm{O}=$ Orédon; $\mathrm{Ll}=$ Lladure.

La dernière colonne récapitule les citations bibliographiques antérieures (numérotées), les synonymies nouvelles, les déterminations erronées ainsi que certaines récoltes, plus fragmentaires dans d'autres points de la chaîne.

Le nombre d'espèces par sous-familles ou tribus est récapitulé pour ces six stations dans le tableau I.

Pour ce qui concerne les espèces de Catalogne espagnole nous reprenons simplement la liste parue dans les trois dernières publications de Prat (1977, 1979 et 1980). La liste primitivement communiquée par Cet auteur (in litt.) comportait quelques citations d'espèces supplémentaires qui ont été supprimées. 


\section{Z: $\mathrm{Ni}$ Li A E $\mathrm{O}$ Ll Citations antérieures (),} autres récoltes

Tanypodinae (23 espèces)

Ablabesmyia longistyla Fitt.

Ablabesmyia monilis (L.)

Apsectrotanypus trifascipennis

(Zett.)

+Conchapelopia melanops (Wied.)

Conchapelopia pallidula (Mg.)

Macropelopia goetghebueri (K.)

Macropelopia nebulosa (Mg.)

Macropelopia notata (Mg.)?

Monopelogia tenuicalcar (K.)

Natarsia punctata (Fabr.)

Nilotanypus dubius (Mg.)

Paramerina divisa (Walk.)

Procladius choreus (Mg.)

Procladius parvulus $\mathrm{K}$.

Psectrotanypus varius (Fabr.)

Thienemannimyia carnea (Fabr.)

Thienemannimyia festiva Fitt.

Thienemannimyia geijskesi (G.)

Thienemannimyia laeta (Mg.)

Thienemannimyia northumbrica (Edw.)

Trissopelopia longimana (Staeg.)

Zavrelimyia barbatipes (K.)

Zavrelimyia melanura (Mg.)

Diamesinae (25 espèces)

Boreoheptagia legeri (G.)

Diamesa aberrata (Lundb.)

Diamesa bertrami Edw.

Diamesa cinerella (Mg.)

Diamesa hamaticornis $\mathrm{K}$.

Diamesa incallida (Walk.)

Diamesa laticauda Ser.-Tos.

Diamesa latitarsis (G.)

Diamesa lavillei Ser.-Tos.

Diamesa steinböcki (G.)

+Diamesa thienemanni K.

Diamesa thomasi Ser.-Tos.

Diamesa wïlkeri Ser.Tos.

Diamesa zernyi Edw.

Monodiamesa ekmani $\mathrm{Br}$.

Pseudokiefferiella parva (Edw.)

+Pothastia gaedii (Mg.)

+Potthastia iberica Ser.-Tos.

Potthastia longimana $\mathrm{K}$.

Prodiamesa olivacea (Mg.)

+Pseudodiamesa branickii (Nov.)

Pseudodiamesa nivosa G.

+ Sympotthastia spinifera Ser.-Tos.

Syndiamesa hygropetrica (K.)

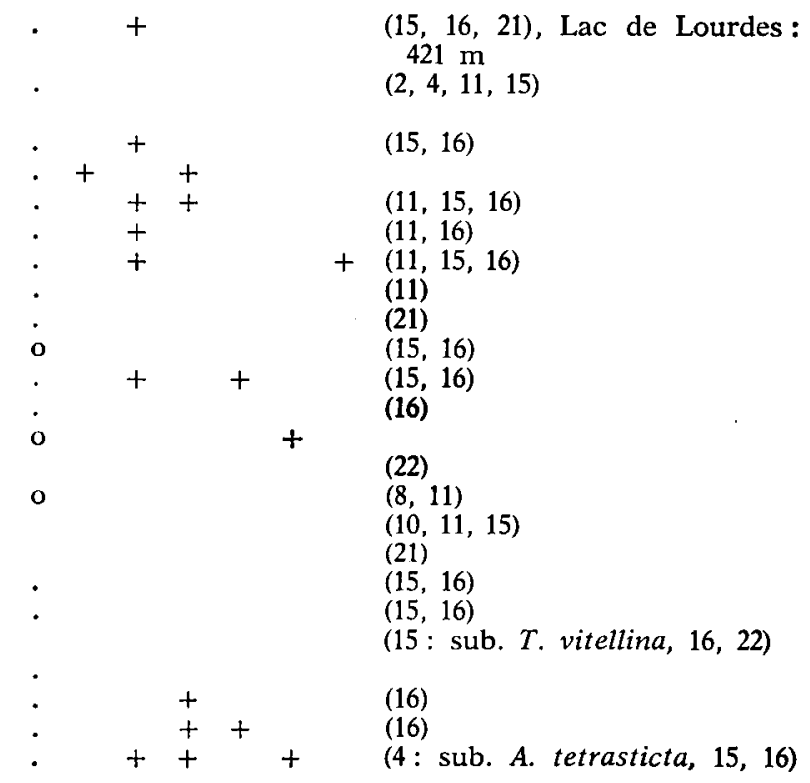

(1, 2, 4, 5, 7, 8 sub. H. punctu(29)

$+++(11,15,29)$

$+++(15,29)$

$(15,29)$

(29)

$+\quad(11,29)$
$+\quad(5-7-11$ sub. D. tonsa, 29)

$(2,3,4,5,7,8,11,29)$

$+$

$(29)$
$+\quad(29)$

$+\quad+\quad$ (15 sub. D. bohemani G., 29)

$+\quad$ (4-5 sub. D. parva)

+
+
$+\quad(4,5,7,11,29)$

$+++(4,5,8,11,15,16,21)$

$++t$

$(8,15,16,29)$, Lac Tourrat: $2621 \mathrm{~m}$

(29) 

autres récoltes

Syndiamesa edwardsi (G.)

Orthocladilinae (104 espèces)

Brillia longifurca $\mathbf{K}$.

Brillia modesta (Mg.) ++ Bryophaenocladius flexidens $\mathrm{Br}$. ++ Bryophaenocladius subvernalis (Edw.)

+ Camptocladius stercorarius (de Geer) + Chaetocladius dentiforceps (Edw.) ++ Chaetocladius excerptus (Walk)

+ Chaetocladius laminatus $\mathrm{Br}$.

++ Corynoneura fittkaui Schlee

Corynoneura lacustris Edw.

Corynoneura lobata Edw.

Corynoneura scutellata Winn.

Cricotopus annulator $\mathrm{G}$.

+ Cricotopus bicinctus $\mathrm{Mg}$.

+Cricotopus fuscus $\mathrm{K}$.

Cricotopus pirifer Hirv.

Cricotopus speciosus $\mathrm{G}$.

Cricotopus sylvestris (Fabr.)

+ Cricotopus tremulus $L$.

Cricotopus triannulatus G.

Cricotopus tricinctus $\mathrm{Mg}$.

Cricotopus trifasciatus $\mathbf{M g}$.

Epoicocladius flavens (Mall)

Eukiefferiella brevicalcar (K.)

Eukiefferiella calvescens Edw.

Eukiefferiella claripennis (Lundb.)

Eukiefferiella coerulescens (K.)

+ Eukiefferiella clypeata (K.)

Eukiefferiella cyanea Th.

Eukiefferiella devonica (Edw.)

Eukiefferiella fittkaui Lehm.

Eukiefferiella fuldensis Lehm.

Eukiefferiella gracei (Edw.)

Eukiefferiella minor Edw.

+Eukiefferiella similis G.

Eukiefferiella tirolensis G.

+ Eukiefferiella veralli Edw.

++ Gymnometriocnemus brumalis (Edw.)

Heleniella ornaticollis (Edw.)

Heterotrissocladius marcidus (Walk.)

Krenosmittia boreoalpina G

++ Limnophyes interruptus G.

+Limnophyes gurgicola Edw.

Limnophyes minimus (Mg.)

+ Limnophyes prolongatus (K.)

Limnophyes smolandicus $\mathrm{Br}$.

Limnophyes truncorum G.

Metriocnemus fuscipes (Mg.)

++ Metriocnemus gracei Edw.

Metriocnemus hirticollis (Staeg.)
(15 sub. Sympotthastia edwarsi, 21 sub. Parapotthastia)

$$
\begin{aligned}
& +\quad+\quad+\text { (15) Lac de Lourdes: } 421 \mathrm{~m} \\
& + \\
& + \\
& +\underset{+}{+}+ \\
& +(16,21) \\
& +\quad+\quad \text { (15 sub. C. kiefferi G., 16) } \\
& \begin{array}{l}
++(15,16) \\
+++\quad(1,2,6,11,16 \text { sub. Trichocla. }
\end{array} \\
& \text { dius alpestris } \mathrm{G} ., 22) \\
& + \\
& + \\
& +\quad+(11,16) \\
& \text { (21) } \\
& +\quad(16,21) \\
& ++++(21) \\
& +++(5,7,11) \\
& \begin{aligned}
+ & (5,7,11) \\
& (2,3,5,7,8,11)
\end{aligned} \\
& ++++ \text { (16) } \\
& ++ \\
& \begin{array}{ll} 
& + \\
? & + \\
+ & +
\end{array} \\
& \begin{array}{r}
+ \\
+\quad+ \\
+\quad(21)
\end{array} \\
& ++(15,1621) \\
& +\quad+\quad(3,11,16) \\
& +\quad++ \text { (15) } \\
& +\quad+\quad \text { (16) } \\
& \begin{array}{l}
+ \\
+\quad+\quad(11,15,21)
\end{array}
\end{aligned}
$$



$\mathrm{Z}_{2} \quad \mathrm{Ni} \quad \mathrm{Li}$ A $\quad \mathrm{E} \quad \mathrm{O} \quad \mathrm{Ll} \quad \begin{gathered}\text { Citations antérieures (), } \\ \text { autres récoltes }\end{gathered}$

Metriocnemus hygropetricus $\mathrm{K}$.

+ Microcricotopus bicolor (Zett.)

Microcricotopus parvulus (K.)

Microcricotopus rectinervis (K.)

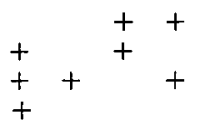

$(2,11,16)$

(16)

\section{ORTHOCLadius}

+ Eudactylocladius bipunctellus

(Zett.)

Eudactylocladius obtexens $\mathbf{B r}$.

Eudactylocladius tuberculatus Edw.

+ Euorthocladius rivicola (K.)

+ Euorthocladius rivulorum (K.)

+ Enorthocladius thienemanni (K.)

++ Orthocladius decoratus (Holm.)

++ Orthocladius dentifer $\mathrm{Br}$.

+Orthocladius excavatus $\mathrm{Br}$.

Orthocladius frigidus (K.)

+Orthocladius oblidens (Walk.)

++ Orthocladius rhyacobius (K.)

+Orthocladius rubicundus (Mg.)

+Orthocladius saxicola (K.)

+Orthocladius smolandicus $\mathrm{Br}$.

Paracladius alpicola (Zett.)

+ Paracladius conversus (Walk.)

+ Paracricotopus niger $\mathrm{K}$.

++ Paracricotopus uliginosus $\mathbf{B r}$

Parakiefferiella bathophila (K.)

Parakiefferiella coronata (Edw.)

Parametriocnemus stylatus (K.)

+ Paraphaenocladius penerasus (Edw.)

Paratrichocladius skirwithensis (Edw.)

Paratrichocladius rufiventris (Mg.) ++ Parorthocladius nudipennis (K.)

+ Parorthocladius torrentium (G.)

Psectrocladius barbatipes $\mathrm{K}$.

Psectrocladius barbimanus (Edw.)

Psectrocladius obvius (Walk.)

Psectrocladius octomaculatus Wülk.

Psectrocladius oligosetus Wülk.

Psectrocladius sordidellus (Zett.)

+ Pseudorthocladius curtistylus G.

+ Pseudosmittia gracilis (G.)

Pseudosmittia trilobata (Edw.)

Rheocricotopus effusus (Walk.)

Rheocricotopus fuscipes (Walk.)

+ Rheocricotopus foveatus Edw.

Smittia aterrima (Mg.)

++ Smittia leucopogon (Mg.)

++ Smittia nudipennis G.

+ Smittia pratorum G.

Symbiocladius rhithrogenae

K. et Zav.

Synorthocladius semivirens (K.) ++ Thienemannia gracilis (K.)

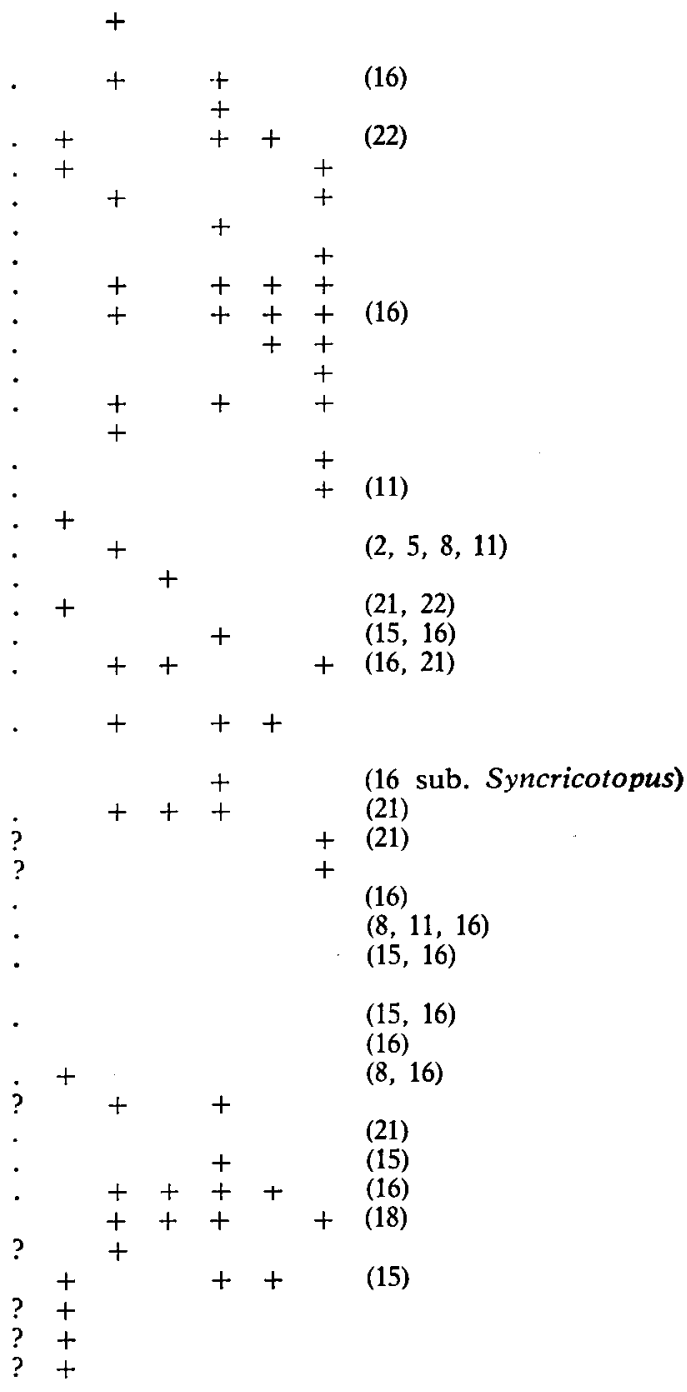

(5)

$+\quad+\quad(2,5,11,16)$
+ 
$\mathrm{Z}_{2} \mathrm{Ni} \quad \mathrm{Li}$ A $\mathrm{E} \quad \mathrm{O}$ Ll $\quad$ Citations antérieures (), autres récoltes

Thienemannia clavicornis $\mathrm{K}$. ++ Thienemanniella flavescens Edw. ++ Thienemanniella lutea Edw. + Thienemanniella obscura $\mathrm{Br}$.

Chironominae (83 espèces)

Chironomini (39 espèces)

+Chironomus anthracinus Zett.

+Chironomus cingulatus (Mg.)

Chironomus commutatus Str.

Chironomus plumosus L.

+Chironomus thummi K.

Cryptocladopelma laccophila $\mathbf{K}$.

Cryptocladopelma lateralis G. ++ Cryptotendipes pseudotener G.

+ Demicryptochironomus vulneratus (Zett.)

+Dicrotendipes notatus (Mg.)

Dicrotendipes pulsus Walk.

Dicrotendipes tritomus $\mathrm{K}$.

Einfeldia dissidens (Walk.)

+ Endochironomus tendens Fabr.

+Glyptotendipes gripekoveni K.

++ Leptochironomus laminata $\mathrm{K}$.

Microtendipes britteni $\mathrm{Edw}$.

Microtendipes confinis (Mg.)

Microtendipes pedellus (de Geer)

Microtendipes rydalensis (Edw.)

Pagastiella orophila (Edw.)

Paracladopelma camptolabis $\mathbf{K}$.

+ Paratendipes albimanus (Mg.)

Pentapedilum nubens Edw.

Pentapedilum tritum (Walk.)

Pentapedilum sordens (v.d.W.)

+Phaenopsectra flavipes (Mg.)

+ Polypedilum acutum K.

Polypedilum albicorne (Mg.)

+Polypedilum convictum (Walk.)

+Polypedilum cultellatum G.

Polypedilum laetum (Mg.)

Polypedilum nubeculosum (Mg.)

+Polypedilum pedestre (Mg.)

+ Polypedilum pullum (Zett.)

Polypedilum scalaenum Schr.

+ Polypedilum vetterense $\mathrm{Br}$.

+ Stenochironomus gibbus Fabr.

Stictochironomus histrio (Fabr.)

TaNytarsini (44 espèces)

Cladotanytarsus atridorsum (K.)

Cladotanytarsus iucundus Hirv.

+ Cladotanytarsus mancus (Walk.) Edw.

Krenopsectra fallax Reiss

Lauterbornia coracina $\mathbf{K}$.

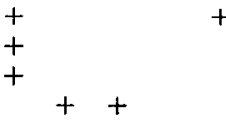

$$
\begin{aligned}
& +\quad+\quad(16) \\
& \begin{array}{ll}
+ & (16,21)
\end{array} \\
& +\quad(8)
\end{aligned}
$$

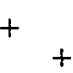

Lac de Lourdes : $421 \mathrm{~m}$

(21 sub. P. quadriguttatum K., 23)

$(16,21)$, Lac de Lourdes : $421 \mathrm{~m}$

$+\quad(20)$
$+\quad+\quad(15,16)$




\section{Z, Ni $\mathrm{Li}$ A $\quad \mathrm{E} \quad \mathrm{O} \quad \mathrm{Ll} \quad$ Citations antérieures (),} autres récoltes

Lithotanytarsus emarginatus G. ++ Micropsectra apposita (Walk.) Micropsectra atrofasciata K. Micropsectra attenuata Reiss Micropsectra bidentata G. Micropsectra contracta Reiss Micropsectra fusca Meig.

+ Micropsectra junci (Meig.) Micropsectra lindrothi $\mathrm{G}$.

Micropsectra notescens (Walk.) Micropsectra recurvata G. Neozavrelia fuldensis Fitt.

Paratanytarsus austriacus $\mathrm{K}$.

Paratanytarsus confusus Pal.

+ Paratanytarsus inopertus (Walk.) Edw.

Paratanytarsus laccophilus Edw.

Paratanytarsus lauterborni $\mathrm{K}$. + Rheotanitarsus curtistylus G.

+ Rheotanytarsus distinctissimus $\mathrm{Br}$.

+ Rheotanytarsus nigricauda Fitt.

+ Rheotanytarsus photophilus G. ++ Stempellina bausei (K.) Edw.

+ Stempellinella brevis Edw.

+ Tanytarsus arduennensis G.

Tanytarsus bathophilus (K.)

Tanytarsus brundini Lind.

Tanytarsus debilis (Mg.)

+Tanytarsus ejuncidus Walk.

+ Tanytarsus eminulus Walk.

Tanytarsus fimbriatus Reiss et Fitt.

+ Tanytarsus heusdensis G.

Tanytarsus holochorus Edw.

Tanytarsus lugens $\mathrm{K}$.

Tanytarsus miriforceps $\mathrm{K}$.

Tanytarsus nemorosus Edw.

+Tanytarsus pallidicornis Walk.

+ Tanytarsus signatus v.d.W.

++ Tanytarsus sylvaticus v.d.W.

+ Zavrelia pentatoma K.
$(1,4,6,7,8,11)$

(21)

$(1,2,6,11)$

(16)

(15)

$\begin{array}{ll}+ & + \\ + & +\end{array}$

(11, 15, 16 sub. M. foliata Lav., 28)

$(15,16)$

$(6,8,11$ sub. $N$. pyrenaea Fitt., $16,21)$

$(2,4,5,7,8,11,16)$

$(14,21)$

$(14,21)$

$(2,5,11$ sub. $P$. boreoalpinus Th., 16)

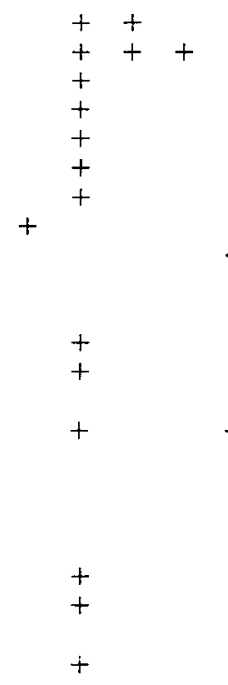

Rivière Le Volp à Sainte-Croix $+\quad(16,27)$

$(15,16,27)$

(14 sub. T. samboni, 16, 27.

$(16,22)$

$+\quad$ (15 sub. $T$. arduennensis)

$(15,16,27)$

$(15,16,21,27)$

$(15,16,27)$

(23)

Lac de Lourdes : $421 \mathrm{~m}$

Tablead I. - Nombre d'espèces de Chironomides de chaque sous-familles ou tribus récoltées dans 6 stations pyrénéennes: $\mathrm{Ni}=$ Nivelle, $\mathrm{Li}=$ Lissuraga, $\mathrm{A}=$ Agos, $\mathrm{E}=$ Estaragne, $\mathrm{O}=$ Orédon, $\mathrm{Ll}=$ Lladure.

\begin{tabular}{lrrrrrr}
\hline & $\mathrm{Ni}$ & $\mathrm{Li}$ & $\mathrm{A}$ & $\mathrm{E}$ & $\mathrm{O}$ & $\mathrm{Ll}$ \\
\hline Tanypodinae & 1 & 7 & 5 & 2 & 2 & 1 \\
Diamesinae & 0 & 3 & 1 & 13 & 7 & 8 \\
Orthocladiinae & 17 & 40 & 15 & 40 & 20 & 26 \\
Chironomini & 5 & 13 & 5 & 1 & 3 & 2 \\
Tanytarsini & 4 & 18 & 3 & 5 & 6 & 1 \\
Total & 27 & 81 & 29 & 61 & 38 & 38 \\
\hline
\end{tabular}




\section{REMARQUES}

- Natarsia nugax (Walk) est signalée de la Zone 2 de la Limnofauna vraisemblablement à la place de Natarsia punctata (Fabr.).

- Zavrelymyia punctatissima (G.) signalée par Laville (1966) n'est pas incluse dans le catalogue : son identification reste incertaine.

- Micropsectra bodanica Reiss est signalée par erreur dans le premier catalogue (1976) et dans la Limnofauna (Fittkau et al 1978) : il s'agit en fait de Micropsectra junci (Mg.).

\section{IV. - DIAMESINI DES PYRENEES : ETUDE BIOGEOGRAPHIQUE}

Les cours d'eau froids de montagne abritent une faune caractérisiique riche en Diamesinae et c'est dans le crénon qu'ils sont le plus diversifiés.

C'est grâce à l'important travail de Serra-Tosio (1973) que la réparition biogéographique de la principale tribu des Diamesini est actuellement la mieux connue en Europe. C'est par référence à cette étude que nous pouvons envisager une analyse biogéographique des Diamesini pyrénéens actuellement inventoriés.

Trois Diamesinae de la faune pyrénéenne ne se rangent pas dans cette tribu :

- Prodiamesa olivacea (tribu des Prodiamesini) est très largement répandue en Europe et également en Sibérie et Mongolie.

- Monodiamesa ekmani, également de la tribu des Prodiamesini, paraît moins eurytherme et plus orophile puisqu'elle est sigalée des Pyrénées, des montagnes boréales et du nord de la Suède mais aussi des plaines occidentales d'Europe.

- Boreoheptagia legeri (tribu des Heptagyini) présente une répartition à caractère orophile : elle est connue des Pyrénées, des Alpes, des Balkans et du Caucase.

Un premier bilan biogéographique concernant les 22 Diamesini pyrénéens peut être envisagé, relativement à la faune européenne.

- 9 des 10 espèces européennes à répartition boréoalpine sont également présentes dans les Pyrénées, ce sont: Pseudodiamesa braniicki, Pseudodiamesa nivosa, Diamesa aberrata, Diamesa incallida, Diamesa bertrami, Diamesa latitarsis, Diamesa steinböcki, Diamesa thienemanni, Pseudokiefferiella parva. Seule, Diamesa lindrothi, présentant une répartition essentiellement arctique avec quelques stations alpines reliques, n'a pas été retrouvée.

- Des 22 espèces à répartition médio-européenne orophile, 9 sont signalées dans la chaîne pyrénéenne. 
a) 3 avec une large répartition européenne : Diamesa cinerella, Diamesa hamaticornis, Diamesa zernyi.

b) 6 avec une aire médio-européenne réduite : Syndiamesa edwardsi et Syndiamesa hygropetrica à stations rares et disséminées; Diamesa wülkeri, connue des Alpes et des Pyrénées et Diamesa laticauda connue en plus des Carpathes; Diamesa thomasi et Diamesa lavillei cnfin, endémiques pyrénéens.

-4 espèces de types médio-européen non orophile appartiennent aux Diamesini les plus eurythermes et ont surtout été récoltées dans les stations basses de la chaîne (Lissuraga), il s'agit de Potthastia gaedii et Potthastia longimanus cette dernière avec une aire de répartition la plus vaste.

Potthastia iberica seulement connue de moyenne altitude de la Sierra Nevada (Espagne) et Sympotthastia spinifera, signalée seulement d'une station de basse altitude $(70 \mathrm{~m})$ de la vallée du Rhône.

La faune pyrénéenne des Diamesini paraît actuellement moins diversifiée que celle des zones montagneuses d'Europe centrale et méridionale (Alpes notamment). En effet, sur les 40 espèces connues de ces zones, seulement 22 y sont répertoriées.

Toutefois, au niveau des espèces boréoalpines présentes dans les eaux froides des altitudes élevées on a pu trouver une faune identique en raison d'une bonne investigation de ces milieux lotiques froids. Les régions de moyenne altitude, par contre, ont été moins bien prospectées ce qui peut expliquer la diversité moindre au niveau des espèces de type médio-européen orophile.

\section{V. - CONCLUSION}

L'ensemble de ces récoltes porte à 235 le nombre des Chironomides recensés actuellement dans les Pyrénées. Ils se répartissent en 23 Tanypodinae, 25 Diamesinae, 104 Orthocladiinae, 39 Chironomini et 44 Tanytarsini.

TABLEAU II. - Répartition des espèces pyrénéennes dans les différentes sousfamilles ou tribus.

\begin{tabular}{lcccccc}
\hline & $\begin{array}{c}\text { Tanypo- Diame- } \\
\text { dinae }\end{array}$ & $\begin{array}{c}\text { Orthocla- } \\
\text { sinae }\end{array}$ & diinae & $\begin{array}{c}\text { Chirono- Tany- } \\
\text { mini }\end{array}$ & $\begin{array}{c}\text { Tarsini } \\
\text { tars }\end{array}$ & Total \\
\hline $\begin{array}{l}\text { Espèces } \\
\text { antérieurement recensées }\end{array}$ & 22 & 20 & 57 & 21 & 26 & 146 \\
$\begin{array}{c}\text { Nombre d'espèces nouvelle- } \\
\text { ment citées des Pyrénées }\end{array}$ & 1 & 5 & 29 & 16 & 15 & 66 \\
$\begin{array}{c}\text { Nombre d'espèces nouvelle- } \\
\text { ment citées de France }\end{array}$ & 23 & 25 & 104 & 39 & 44 & 235 \\
\begin{tabular}{l} 
Total des espèces \\
\hline
\end{tabular} & 23 & & 18 & 2 & 3 & 23 \\
\hline
\end{tabular}


D'un point de vue faunistique, il s'agit pour 89 espèces $(+)$ de premières citations dans les Pyrénées et pour 23 d'entre elles $(++)$ de premières récoltes en France (tableau II). C'est surtout dans la sousfamille des Orthocladiinae que l'on trouve le plus d'espèces nouvellement citées (47) en raison de la prospection plus intensive de stations lotiques, les milieux lacustres naturels de moyenne et basse aititude s'avérant en fait plus rares dans l'ensemble du massif pyrénéen.

\section{TRAVAUX CITES}

[1] Bertrand (H.). 1950 a. - Chironomides pyrénéens nouveaux pour la faune française. Bull. Soc. ent. Fr., 55: 11-12.

[2] Bertrand (H.). 1950 b. - Diptères Chironomides pyrénéens. Bull. Soc. ent. Fr., $55: 153-155$.

[3] Bertrand (H.). $1950 \mathrm{c}$. - Récoltes de Diptères Chironomides dans les Pyrénées. Vie et Milieu, 1 (3) : 345-355.

[4] Bertrand (H.). 1952. - Récoltes de Diptères Chironomides dans les Pyrénées. ( $2^{\circ}$ note). Vie et Milieu, 3 (3) : 314-321.

[5] Bertrand (H.). 1953. - Diptères Chironomides pyrénéens (2 note). Bull. Soc. ent. Fr., $58:$ 76-79.

[6] Bertrand (H.). 1955 a. - Sur la faune entomologique des Pyrénées. Verh. Int. Ver. Limnol., 12 : 634-642.

[7] Bertrand (H.). 1955 b. - A propos de tufs à Chironomides. L'entomologiste, $\mathrm{XI}, 1: 6-7$.

[8] Bertrand (H.). 1956. - Diptères Chironomides pyrénéens et espagnols. Bull. Soc. ent. Fr., 61 : 93-95.

[9] Dumas (J.) et Lavandier (P.). 1970. - Etude écologique de la faune aquatique de la cressonnière d'Agos (Hautes-Pyrénées) : cycles de développement, répartition. Thèse $3^{*}$ cycle. Toulouse : $158 \mathrm{p}$.

[10] Fittkau (E. J.). 1962. - Die Tanypodinae (Dipt. Chironomidae). Abh. Larvalsyst. Insekten, $6: 1-453$.

[11] Fittkau (E. J.), Schlee (D.) et Reiss (F.). 1967. - Chironomidae in Illies (J.), Limnofauna Europaea. Gustav Fischer Verlag, Stuttgart : 346-381.

[11 a] FittKau (E. J.), SchleE (D.) et Reiss (F.). 1978. - Chironomidae in Illies (J.), Limnofauna Europaea. Gustav Fischer Verlag, Stuttgart : 404-440.

[12] Lavandier (P.). 1974. - Ecologie d'un torrent pyrénéen de haute montagne. I. Caractéristiques physiques. Annls Limnol., 10 (2) : 173-219.

[13] Lavandier (P.) et MUR (C.). 1974. - Ecologie d'un torrent pyrénéen de haute montagne. II. Caractéristiques chimiques. Annls. Limnol., 10 (3) : 275-309.

[14] Laville (H.). 1965. - Micropsectra foliata n. sp. Une nouvelle espèce de Chironomidae. Annls Limnol., 1 (1) : 73-81.

[15] Laville (H.). 1966. - Chironomides du massif de Néouvielle (Pyrénées centrales) (Diptères). Annls Limnol., 2 (1) : 203-216.

[16] Laville (H.). 1972 (1971). - Recherches écologiques sur les Chironomides (Diptera) des lacs du Massif de Néouvielle (Hautes-Pyrénées). Première partie : systématique, écologie, phénologie. Annls Limnol., 7 (2) : 173-332.

[17] Laville (H.) et Lavandier (P.). 1977. - Les Chironomides (Diptera) d'un torrent pyrénéen de haute montagne: l'Estaragne. Annls Limnol., 13 (1) : 57-81.

[18] LehmanN (J.). 1969. - Die europäischen Arten der Gattung Rheocricotopus und drei neue Artvertreter dieser Gattung aus der Orientalis (Diptera, Chironomidae). Arch. Hydrobiol., 66 : 348-369. 
[19] Masson (D.). 1977. - Contribution à l'étude des apports d'organismes aériens en milieu lacustre. Thèse $3^{\mathrm{e}}$ cycle, Univ. P.-Sabatier, Toulouse : $134 \mathrm{p}$.

[20] Neveu (A.). 1972. - Introduction à l'étude de la faune des Diptères à larves aquatiques d'un ruisseau des Pyrénées-Atlantiques, le Lissuraga. Ann. Hydrobiol, 3 (2) : 173-196.

[21] Prat (N.). 1977. - Quironomidos de Cataluña (Diptera). Graellsia, 31 : 157-185.

[22] Prat (N.). 1979. - Quironomidos de los embalses españoles. Graellsia, 33 : $37-96$.

[23] Prat (N.). 1980. - Quironomidos de los embalses españoles. Graellsia, 34 (1978) : 59-119.

[24] Pujol (J. Y.). 1975. - Quelques aspects de la biologie des Drusinae (Trichoptères) et influences humaines sur un cours d'eau de montagne. Thèse $3^{\mathrm{e}}$ cycle, Univ. P.-Sabatier, Toulouse : 133 p. et 19 p. annexes.

[25] ReIss (F.). 1969. - Krenopsectra fallax gen. n. sp. n. (Diptera, Chironomidae) aus den Alpen und Pyrenäen. Ann. Zool. Fennici, 6 : 435-442.

[26] ReIss (F.). 1974. - Revision des Typen-Materials einiger Tanytarsini-Arten (Chironomidae, Diptera) aus dem Museum Brüssel. Ent. Tidskr., 95, Suppl. : 203-211.

[27] Reiss (F.) et Fitrkau (E. J.). 1971. - Taxonomy and ecology of European distributed Tanytarsus-species (Chironomidae, Diptera). Arch. Hydrobiol., Suppl. $40: 75-300$.

[28] SäwEDAL (L.). 1976. - Revision of the notescens-group of the genus Micropsectra Kieffer, 1909 (Diptera: Chironomidae). Ent. Scand., 7 : 109-144.

[29] Serra-Tosio (B.). 1973. - Ecologie et biogéographie des Diamesini d'Europe (Diptera, Chironomidae). Trav. Lab. Hydrobiol. Grenoble, 63, année 1971 : 5-175.

[30] Thibault (M.). 1971. - Ecologie d'un ruisseau à truites des Pyrénées-Atlantiques, le Lissuraga. I. Etude critique du milieu. Ann. Hydrobiol., 2 (2) : 209239. 